\title{
Repeated Double-Poling Sprint Training in Hypoxia by Competitive Cross-country Skiers
}

\author{
RAPHAEL FAISS ${ }^{1,2}$, SARAH WILLIS ${ }^{3}$, DENNIS-PETER BORN ${ }^{4}$, BILLY SPERLICH ${ }^{3,4}$, JEAN-MARC VESIN $^{5}$, \\ HANS-CHRISTER HOLMBERG ${ }^{3}$, and GRÉGOIRE P. MILLET ${ }^{1,2}$
}

${ }^{1}$ Institute of Sport Sciences, University of Lausanne, Lausanne, SWITZERLAND; ${ }^{2}$ Department of Physiology, Faculty of Biology and Medicine, University of Lausanne, Lausanne, SWITZERLAND; ${ }^{3}$ Nationellt Vintersport Centrum, Swedish Winter Sports Research Centre, Mid Sweden University, Östersund, SWEDEN; ${ }^{4}$ Department of Sport Science, University of Wuppertal, Wuppertal, GERMANY; and ${ }^{5}$ Applied Signal Processing Group, Swiss Federal Institute of Technology, Ecole Polytechnique Fédérale de Lausanne, Lausanne, SWITZERLAND

\begin{abstract}
FAISS, R., S. WILLIS, D.-P. BORN, B. SPERLICH, J.-M. VESIN, H.-C. HOLMBERG, and G. P. MILLET. Repeated Double-Poling Sprint Training in Hypoxia by Competitive Cross-country Skiers. Med. Sci. Sports Exerc., Vol. 47, No. 4, pp. 809-817, 2015. Purpose: Repeated-sprint training in hypoxia (RSH) was recently shown to improve repeated-sprint ability (RSA) in cycling. This phenomenon is likely to reflect fiber type-dependent, compensatory vasodilation, and therefore, our hypothesis was that RSH is even more beneficial for activities involving upper body muscles, such as double poling during cross-country skiing. Methods: In a double-blinded fashion, 17 competitive cross-country skiers performed six sessions of repeated sprints (each consisting of four sets of five 10-s sprints, with 20-s intervals of recovery) either in normoxia ( $\left.\mathrm{RSN}, 300 \mathrm{~m} ; \mathrm{F}_{\mathrm{i}} \mathrm{O}_{2}, 20.9 \% ; n=8\right)$ or normobaric hypoxia $\left(\mathrm{RSH}, 3000 \mathrm{~m} ; \mathrm{F}_{\mathrm{i}} \mathrm{O}_{2}, 13.8 \% ; n=9\right)$. Before (pre) and after (post) training, performance was evaluated with an RSA test (10-s all-out sprints-20-s recovery, until peak power output declined by $30 \%$ ) and a simulated team sprint (team sprint, $3 \times 3$-min all-out with 3 -min rest) on a double-poling ergometer. Triceps brachii oxygenation was measured by near-infrared spectroscopy. Results: From pretraining to posttraining, peak power output in the RSA was increased $(P<0.01)$ to the same extent $(29 \% \pm 13 \%$ vs $26 \% \pm 18 \%$, nonsignificant $)$ in RSH and in RSN whereas the number of sprints performed was enhanced in RSH $(10.9 \pm 5.2$ vs $17.1 \pm 6.8, P<0.01)$ but not in $\operatorname{RSN}(11.6 \pm 5.3$ vs $11.7 \pm 4.3$, nonsignificant). In addition, the amplitude in total hemoglobin variations during sprints throughout RSA rose more in RSH $(P<0.01)$. Similarly, the average power output during all team sprints improved by $11 \% \pm 9 \%$ in RSH and $15 \% \pm 7 \%$ in RSN. Conclusions: Our findings reveal greater improvement in the performance of repeated double-poling sprints, together with larger variations in the perfusion of upper body muscles in RSH compared with those in RSN. Key Words: ALTITUDE TRAINING, REPEATED SPRINTS, CROSS-COUNTRY SKI, PERFORMANCE
\end{abstract}

$\mathrm{F}$ or many years now, coaches and athletes have combined the stress of training with hypoxia to elicit even greater physiological adaptations $(12,15,27,40,42)$ initially by combining hypoxia with low-intensity aerobic exercise to enhance oxygen transport and, more recently, with high-intensity interval training or repeated sprints.

However, a recent review concludes that the additional benefits for sea-level performance of intermittent hypoxic training (IHT) compared with those of similar training under normoxic conditions are strikingly small (14). At the same time, three interesting investigations reveal that a novel approach involving repeated-sprint training in hypoxia (RSH)

Address for correspondence: Raphael Faiss, Ph.D., Department of Physiology, Faculty of Biology and Medicine, Institute of Sport Sciences, University of Lausanne, Geopolis, 1015 Lausanne, Switzerland; E-mail: Raphael.Faiss@unil.ch. Submitted for publication May 2014.

Accepted for publication July 2014.

0195-9131/15/4704-0809/0

MEDICINE \& SCIENCE IN SPORTS \& EXERCISE ${ }_{\circledast}$

Copyright (C) 2014 by the American College of Sports Medicine

DOI: 10.1249/MSS.0000000000000464 does provide additional systematic benefits in comparison with the same training under normoxic conditions $(15,17,33)$.

Because RSH is based on repetition of "all-out" efforts of short ( $\leq 30 \mathrm{~s})$ duration separated by short periods of incomplete recovery, the efficiency of this strategy probably depends on the maximal intensity of the successive bouts of sprinting (separated by very short periods of recovery) to evoke potent adaptations at the molecular level and, possibly, in the delivery of oxygen $\left(\mathrm{O}_{2}\right)$ to the mitochondria (15). Indeed, the intensity of hypoxic training per se seems to modulate muscle performance at the molecular level with "adaptations that compensate for the reduced availability of oxygen during exercise" (25). Moreover, the limitation of $\mathrm{O}_{2}$ delivery during exercise triggers compensatory vasodilation, increasing blood flow in an attempt to maintain sufficient delivery of $\mathrm{O}_{2}$ to muscles to an extent dependent on the intensity of the exercise (10).

Repeated sprints under hypoxic conditions may benefit from such adaptive mechanisms, perhaps stimulating the diffusion of $\mathrm{O}_{2}$ into working muscles $(10,15)$. We have reported that the repeated-sprint ability (RSA) of trained cyclists improved significantly after as few as eight sessions of RSH (15). In that study, the additional benefit of RSH 
was thought to involve improved $\mathrm{O}_{2}$ extraction by the fasttwitch fibers (FT) (which are those predominantly recruited during sprints (22)) as a result of the high-intensity repeated sprinting under hypoxic conditions (28). Greater amplitudes of muscle blood perfusion variations suggesting enhanced muscle blood flow supported this hypothesis of greater $\mathrm{O}_{2}$ use by FT after RSH.

Accordingly, physical activity involving extensive recruitment of FT may benefit the most from RSH. Of interest in this context is the fact that upper arm muscles contain a high proportion of FT (26). For example, in professional tennis players, two-thirds of all the fibers in the $m$. triceps brachii are FT, whereas the corresponding value for the $m$. vastus lateralis is only one-third (34). Moreover, in elite cross-country (XC) skiers, the proportions of FT were also shown to be higher in the triceps brachii than those in the vastus lateralis muscles (32).

Consequently, if RSH improves the extraction of $\mathrm{O}_{2}$ by FT, the benefits seen in trained cyclists should certainly occur in XC skiers as well. During double-poling XC skiing, upper body muscles contribute substantially to power production (24), and even if this sport is thought to be primarily aerobic, recent developments include the introduction of shorter racing formats, such as individual and team sprints. Such events require greater anaerobic capacity and upper body power (35) and, together with the repeated changes in velocity and intermittent sprints that occur during mass start races, underline the decisive role of sprinting ability for the outcome of a competition. Clearly, improving RSA may give $\mathrm{XC}$ skiers (including top athletes) a competitive edge.

Nonetheless, the potential associated benefits for the ability to repeat sprints under normoxic conditions after RSH or normoxic training (RSN) have never been compared in a randomized, double-blind, controlled study on XC skiers. Such a comparison revealed that repeated-sprint training under hypoxic conditions can improve subsequent performance. Here, XC ski double poling was selected as a model to elucidate the influence of RSH training on the upper body performance of highly trained athletes in a reproducible and controlled manner. Moreover, de-/reoxygenation of the triceps brachii, a muscle group containing a high proportion of FT fibers, was measured to evaluate potential fiber typespecific adaptations triggered by RSH.

We hypothesized that repeated-sprint training in hypoxia improves the performance of competitive $\mathrm{XC}$ skiers more than the same training under normoxic conditions.

\section{METHODS}

\section{Subjects}

Seventeen highly trained XC skiers ( 11 men $(29.6 \pm 8.8 \mathrm{yr}$, $1.82 \pm 0.08 \mathrm{~m})$ and six women $(24.2 \pm 5.0 \mathrm{yr}, 1.68 \pm 0.09 \mathrm{~m}))$ (Table 1) were recruited from Swedish national and regional $\mathrm{XC}(n=15)$ and biathlon teams $(n=2)$. Subjects were familiar to the double-poling ergometer and to high-intensity
TABLE 1. Subjects' characteristics before (pre) and after (post) repeated double-poling sprint training in normoxia (RSN) or in hypoxia (RSH).

\begin{tabular}{|c|c|c|c|c|}
\hline & \multicolumn{2}{|c|}{$\operatorname{RSN}(n=8)$} & \multicolumn{2}{|c|}{ RSH $(n=9)$} \\
\hline & Pretraining & Posttraining & Pretraining & Posttraining \\
\hline Body mass (kg) & $74.7 \pm 7.8$ & $74.7 \pm 7.6$ & $72.2 \pm 7.0$ & $72.3 \pm 7.1$ \\
\hline Hematocrit $(\%)$ & $46.0 \pm 3.2$ & $45.7 \pm 2.3$ & $46.5 \pm 2.0$ & $46.1 \pm 1.8$ \\
\hline Hemoglobin $\left(\mathrm{g} \cdot \mathrm{L}^{-1}\right)$ & $154 \pm 13$ & $152 \pm 15$ & $156 \pm 7$ & $152 \pm 12$ \\
\hline$\dot{\mathrm{V}} \mathrm{O}_{2 \text { peak }}\left(\mathrm{L} \cdot \mathrm{min}^{-1}\right)$ & $4.2 \pm 0.8$ & $4.0 \pm 0.8$ & $4.1 \pm 0.6$ & $4.2 \pm 0.7$ \\
\hline
\end{tabular}

exercise bouts from their usual training practice and competitive background. Subjects were all nonsmokers and lowlanders. None of the subjects were acclimatized or recently exposed to altitude, and they were not exposed to an altitude of more than $500 \mathrm{~m}$ during the protocol. The responses induced in male and female athletes by RSH might differ. However, a recent study (36) reported that "men and women matched for initial-sprint work experience similar levels of fatigue and systemic, cerebral, and peripheral adjustments" during repeated-sprint exercise under both normoxic and hypoxic conditions. Subjects provided their written informed consent after the state medical ethics committee approved the experiment (regional ethical review board, Umeå, Sweden Agreement 2013-72-31 M) performed according to the Declaration of Helsinki.

\section{Study Design}

Experimental protocol consisted in two testing sessions before (pre) and after (post) a specific repeated-sprint training period of $2 \mathrm{wk}$ (three sessions per week). Subjects were assigned into a specific training group, as follows: repeatedsprint training in hypoxia (RSH, $n=9$; six males and three females) or repeated-sprint training in normoxia (RSN, $n=8$; five males and three females).

\section{Performance Tests}

RSA test. RSA was tested with 10 -s all-out doublepoling sprints repeated with incomplete recoveries of $20 \mathrm{~s}$ until task failure.

Subjects first performed a 4-min 50-s aerobic warm-up at $1 \mathrm{~W} \cdot \mathrm{kg}^{-1}$ followed by an isolated 10 -s all-out sprint, 4-min 50 -s active recovery at $1 \mathrm{~W} \cdot \mathrm{kg}^{-1}$, and a second isolated 10 -s all-out sprint. After $10 \mathrm{~min}$ of passive recovery seated (including 5 min with the right arm kept still to calibrate the near-infrared spectroscopy (NIRS) device), the RSA test started with $1 \mathrm{~min}$ at $1 \mathrm{~W} \cdot \mathrm{kg}^{-1}$ before the first sprint.

Subjects were given very strong verbal encouragement to perform as many sprints as possible until task failure. Peak power was defined as the highest power recorded in association with a single stroke during all RSA sprints (always the first or second sprint). Task failure was then set at $70 \%$ of this peak power. Subjects were given a warning when they did not reach that value the first time and the test was ended the second time. Subjects were not told about the criterion for task failure or any indication on the number of sprints performed. In addition, to avoid any protective pacing 
strategy, subjects were requested to reach at least $95 \%$ of the best peak power of the two isolated sprints during the first two sprints, which was the case in all subjects. Unfinished sprints (subjects not being able to pull anymore) were not taken into consideration.

Team sprint simulation aerobic test. Exactly $45 \mathrm{~min}$ after the end of the RSA test, subjects were asked to warm up again by completing three incremental steps of $4 \mathrm{~min}$ at 1 , 1.5 , and $2 \mathrm{~W} \cdot \mathrm{kg}^{-1}$. Then, after an additional 5 -min passive rest, subjects were asked to perform a team sprint simulation (TS) consisting of three bouts of 3-min all-out interspersed with 3 -min active recovery at $0.5 \mathrm{~W} \cdot \mathrm{kg}^{-1}$. Strong verbal encouragement was given throughout the test, and poling frequency was instructed to be as high as possible at all times to elicit maximal all-out performance. Information about elapsed time was only given during the final minute every $15 \mathrm{~s}$ to prevent pacing and to further motivate subjects. A peak in oxygen consumption $\left(\mathrm{V}_{2 \text { peak }}\right)$ was calculated as the highest 30-s average value during the test. Although residual fatigue from the RSA test was probable, TS was performed for all subjects at the same moment of the testing sequence, allowing for a good comparison of subjects' aerobic capacity (average power of all 3-min bouts).

\section{Equipment and Measured Variables}

A specially designed double-poling ergometer (SkiErg; Concept2, Morrisville, VT) was used for all training sessions and performance tests. The ergometer is equipped with $\mathrm{XC}$ ski handles and straps (Leki, Kirchheim, Germany). Power is produced by pulling cords spinning a wind resistance flywheel. A damper located on the flywheel housing controls the airflow and, thus, the necessary work to accelerate the flywheel during each stroke. The flywheel deceleration rate (called drag factor by the manufacturer) digitally displayed on the ergometer's interface was used to set and reproduce the resistance individually for each test and training session. Drag factor was set at $130 \%$ and $110 \%$ of body weight in male and female subjects, respectively. These levels, selected on the basis of pilot tests, were designed to allow each athlete to perform 10-15 pre-sprints before task failure. To simulate natural XC skiing, doublepoling movement subjects stood with their own XC ski boots in bindings screwed in a wooden plate. The distance between the bindings and the ergometer was freely chosen by the subjects to mimic their habitual position and reproduced for each test and training session. A wide belt around the subjects' hips was additionally attached to the wall behind them with an elastic band to induce some resistance at the hips to better simulate the double-poling sprinting movement (Fig. 1B).

The ergometer displays instantaneous power output and cycle frequency for each stroke that were recorded externally in a spreadsheet (Excel; Microsoft, Redmond, WA) using a Microsoft ActiveX ${ }^{\circledR}$ software component to extract data live. The ergometer's readings of power output during each sprint were displayed purposefully to both the subjects
A
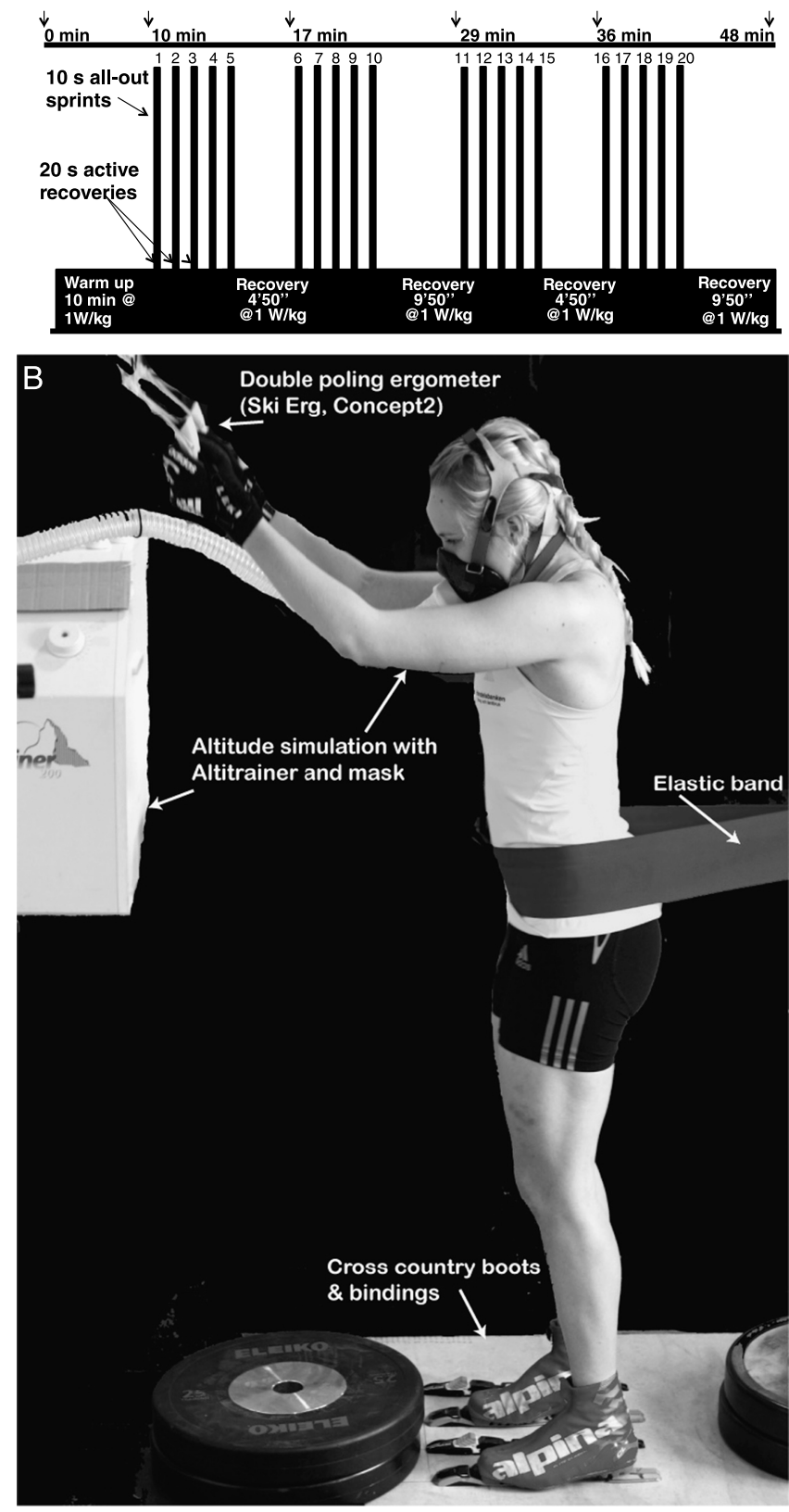

FIGURE 1-A. Description of one training session. B. Ergometer setup for XC ski double poling.

and investigators in an attempt to further motivate optimal performance. Other values such as HR and oxygen saturation were not revealed. $\dot{\mathrm{V}} \mathrm{O}_{2 \text { peak }}$ was determined during TS in pretraining and posttraining with an ergospirometry device (AMIS 2001; Innovision A/S, Odense, Denmark) as the highest 30 -s average measured.

Muscle oxygenation, blood lactate, blood $\mathrm{pH}$, hematocrit, blood hemoglobin concentration, arterial oxygen saturation $\left(\mathrm{SO}_{2}\right), \mathrm{HR}$, and RPE were measured at rest and at the end of each performance test as well as during the first and sixth training sessions (at rest and after the final sprint).

Muscle oxygenation was measured using an NIRS technique as described elsewhere (6). The NIRS device (Portamon; 
Artinis, Zetten, The Netherlands) was used to measure changes in muscle oxygenation by placing a triple optode sensor on the muscle belly of the right arms $m$. triceps brachii with an interoptode spacing of $40 \mathrm{~mm}$. The probe was protected with a thin transparent plastic sheet and then attached to the skin with a double-sided tape and firmly fastened with an opaque cotton elastic band wrapped around the subjects' arms. The position of the probe was marked during pretraining with a permanent pen for accurate repositioning. Differential pathlength factor was set to 3.79 and 4.67 in males and females, respectively (13). All signals were recorded with a sampling frequency of $20 \mathrm{~Hz}$. They were downsampled at $10 \mathrm{~Hz}$ using MATLAB (MATLAB Software, Nattick, MA) routine resample. Then, a 10th-order low-pass zero-phase Butterworth filter (cutoff frequency, $0.1 \mathrm{~Hz}$ ) was applied to the resampled signals to remove possible artifacts and smooth the movement-induced perturbations. Automatic detection of the start and end times of the successive sprints was obtained by estimating the filtered deoxyhemoglobin signal upper and lower envelopes using local minima and maxima in a sliding window of 400 samples length $(4 \mathrm{~s})$. The starting and end times were obtained as the times of contact between the envelopes and the signal. This allowed determination of maximum and minimum for each signal during the successive sprint and recovery phases.

Concentrations for deoxyhemoglobin $([\mathrm{HHb}])$ and total hemoglobin/myoglobin ([tHb]) were recorded. Because $[\mathrm{HHb}]$ values were proposed to be less sensitive to blood flow variations than oxyhemoglobin $\left(\mathrm{O}_{2} \mathrm{Hb}\right)(11)$ and changes in $\mathrm{O}_{2} \mathrm{Hb}$ signals might be confounded by rapid blood volume changes during sprints (7), only $[\mathrm{HHb}]$ and $[\mathrm{tHb}]$ were analyzed for relevant interpretations. Differences between maximum and minimum concentrations were defined as the amplitude of the variation for each sprint $(\Delta[\mathrm{tHb}]$ and $\Delta[\mathrm{HHb}])$, and $\Delta[\mathrm{tHb}]$ was used as an index of blood perfusion (15). Thus, for example, at the beginning of each sprint, a maximum in $[\mathrm{tHb}]$ is observed (i.e., end of each recovery period) and $[\mathrm{tHb}]$ decreases to reach a minimum value at the end of each sprint. The concentration recorded during a 3 -min seated rest with no arm movement was set as $100 \%$ for standardization (Fig. 3). So, amplitudes for each sprint (i.e., $\Delta[\mathrm{tHb}]$ and $\Delta[\mathrm{HHb}]$ ) and the average value for all sprints throughout the RSA test (i.e., $\Delta[\mathrm{tHb}]_{\mathrm{av}}$ and $\Delta[\mathrm{HHb}]_{\mathrm{av}}$ ) were calculated.

Surface EMG signals were recorded from the triceps brachii, latissimus dorsi, rectus abdominis, and soleus muscles from one side of the body (randomized, right, or left) using surface electrodes (Ambu A/S, Ballerup, Denmark), TeleMyo $2400 \mathrm{~T} \mathrm{G}^{\mathrm{TM}}$ data logger (Noraxon, Cologne, Germany), and MyoResearch XP Master Edition ${ }^{\circledR}$ software version 1.08.27 (Noraxon, Scottsdale, AZ). Surface electrodes (Ambu® Blue Sensor N; Ambu A/S, Ballerup, Denmark) were positioned with $20 \mathrm{~mm}$ of interelectrode distance on the prepared skin (impedance, $<5 \mathrm{k} \Omega$ ) after muscle belly identification by palpation of the contracted muscle and marked for proper repositioning after testing. Athletes performed a series of previously practiced 5-s maximal isometric voluntary contractions (MVC) to normalize the data to each individual. Before calculation of EMG variables, all raw signals were processed first to determine the MVC using the IIR filter with a band pass 20- to $400-\mathrm{Hz}$ Butterworth approximation then treated with EMG reduction in the latissimus dorsi channel, followed by RMS smoothing with a window of $100 \mathrm{~ms}$ and amplitude normalization to the peak value with a window of $250 \mathrm{~ms}$. Calculations were performed to assess the mean amplitude expressed in percentage of MVC during both performance tests. Analyses of the RSA included calculation of the average of all cycles performed during each 10-s sprint period. Thirty-second averages were used for the analysis of the TS data.

Blood lactate concentration was measured using a Biosen lactate measurement device (C-Line Sport; EKF Industrial Electronics, Magdeburg, Germany) from $20-\mu \mathrm{L}$ fingertip capillary blood. Single values for blood $\mathrm{pH}$, hematocrit, hemoglobin concentration, and $\mathrm{SO}_{2}$ were assessed by collecting $20-\mu \mathrm{L}$ earlobe capillary blood analyzed immediately in a blood analyser (ABL800 CO-OX Flex; Radiometer, Copenhagen, Denmark). HR was recorded at $1 \mathrm{~Hz}$ by telemetry (RS800; Polar Oy, Kempele, Finland). RPE was assessed for the legs, arms, and breathing using a 6-20 Borg scale (4).

Training Protocol. Training consisted of six sessions of repeated double-poling sprint over 2 wk on an XC ski ergometer including each time four sets of five sprints of $10 \mathrm{~s}$ interspersed with $20 \mathrm{~s}$ of recovery (Fig. 1A). Warm-up and recovery periods between sets were performed with an individualized resistance of $1 \mathrm{~W} \cdot \mathrm{kg}^{-1}$.

Between the first and last two sets, recovery was allowed for $4 \mathrm{~min} 50 \mathrm{~s}$, whereas to promote the highest possible peak power in the final two sets, $9 \mathrm{~min} 50 \mathrm{~s}$ of recovery was allowed between sets 2 and 3 .

For all training sessions, subjects wore a comfortable oronasal mask allowing normal breathing. Subjects breathed either room air (for RSN) or hypoxic air (for RSH) provided via the mask through a hose (suspended to the ceiling by a supportive elastic band) connected with a three-way valve to an altitude simulation device (Altitrainer; SMTech, Nyon, Switzerland). For RSH, ambient air was mixed with nitrogen (from pressurized tanks) and inspired oxygen fraction was reduced to $13.8 \% \pm 0.1 \%$ to simulate an altitude of $3000 \mathrm{~m}$. Two independent researchers assigned subjects randomly to RSN or RSH, with groups matched upon subjects' peak oxygen uptake measured during the TS aerobic test in pretraining. Helpers then handled the altitude simulation device to provide either normal air or hypoxic air during training by turning the three-way valve so that two subjects training simultaneously inspired either air from the altitude simulator or from the room in the well-ventilated laboratory at a constant temperature of approximately $24^{\circ} \mathrm{C}$. This way, the altitude simulator (with the nitrogen outlet sounding) was running during all training sessions. Subjects and investigators were subsequently unable to know whether training was performed in hypoxia or normoxia, and the 
TABLE 2. Parameters measured during supervised double-poling training in the RSN and RSH training groups.

\begin{tabular}{lcc}
\hline & RSN & RSH \\
\hline Total work of all training sessions (kJ) & $1290 \pm 197$ & $1335 \pm 137$ \\
Mean peak power output (W) & $482 \pm 149$ & $481 \pm 131$ \\
Mean HR (bpm) & $149 \pm 11$ & $144 \pm 13$ \\
Mean $\mathrm{SO}_{2}$ after last sprint of each session (\%) & $94.3 \pm 1.3$ & $86.0 \pm 4.2^{*}$ \\
Mean RPE (arms) & $17.6 \pm 0.7$ & $16.9 \pm 1.1$ \\
Mean RPE (legs) & $16.7 \pm 1.4$ & $17.0 \pm 1.8$ \\
Mean RPE (breathing) & $18.3 \pm 0.4$ & $18.0 \pm 1.0$ \\
\hline
\end{tabular}

RPE was measured using Borg 6-20 scale.

${ }^{\star} P<0.01$ for difference with RSN

protocol was run in a double-blind condition. Subjects were told that all training sessions were to be performed at a given altitude without being told exactly which altitude. Subjects were asked to avoid any strenuous training outside the protocol but to keep their usual aerobic training sessions that were reported in a training diary.

Subjects filled a questionnaire reporting their physical activity and food and drink intake during the $48 \mathrm{~h}$ before each testing session. During these $48 \mathrm{~h}$, subjects were asked to refrain from any training or exhaustive activity. All testing sessions were carried out at the same time of the day, starting at 8:00 a.m., and subjects were requested to sleep at least $8 \mathrm{~h}$ the night before each test. Because of the extreme intensity of the tests, subjects were asked not to report to the laboratory on an empty stomach. A standard breakfast (bread with jam and water) was therefore advised. Subjects were asked to replicate the last meals and drinks, avoiding alcohol and caffeine intake during the $24 \mathrm{~h}$ before each test. A preliminary visit allowed subjects to familiarize with the ergometer by completing a 15min trial including $2 \times 3$ repeated sprints of $10 \mathrm{~s}$.

\section{Statistical Analyses}

Data are presented as mean (SD). Performance and blood perfusion changes during RSA test were first evaluated with a two-way (training group-sprint number) general linear model repeated-measures ANOVA with all pairwise multiple comparison procedures (Holm-Sidak method). Performance improvement, muscle oxygenation during RSA, blood lactate, and other single-variable tests were then evaluated with two-way (training group-time (pre- vs posttraining)) general linear model repeated-measures ANOVA with all pairwise multiple comparison procedures (Holm-Sidak method). All analyses were made using Sigmaplot 11.0 software (Systat Software, San Jose CA). Null hypothesis was rejected at $P<0.05$.

\section{RESULTS}

Total work and training intensity during supervised training were similar during RSN and RSH (Table 2). In addition to specific training in the laboratory, subjects completed a similar amount of aerobic training between pretraining and posttraining of $16.6 \pm 6.6$ and $16.3 \pm 6.5 \mathrm{~h}$ in RSH and RSN, respectively. Total hypoxic exposure was $4.8 \pm 0.2 \mathrm{~h}$ in RSH.

RSA performance. Performance results at the pretraining and posttraining are summarized in Table 3. No significant differences between groups were observed in any variable in pretraining. During the RSA test, peak power output $[29 \% \pm$ $13 \%$ vs $26 \% \pm 18 \%$, nonsignificant (NS)] and likewise the average power of all sprints $(18 \% \pm 16 \%$ vs $22 \% \pm 14 \%$, NS) increased $(P<0.01)$ to the same extent from pretraining to posttraining in RSH and in RSN. The number of sprints before exhaustion was increased in RSH (from $10.9 \pm 5.2$ to $17.1 \pm 6.8$, $P<0.01)$ but not in RSN $(11.6 \pm 5.3$ and $11.7 \pm 4.3$, NS $)$ (Fig. 2). In posttraining, compared with that in pretraining, 10 -s peak power in the successive sprints was significantly improved until the 15th sprint in RSH and until the 11th in RSN. Significant group (RSH vs RSN)-time (pretraining vs posttraining) interactions were found in the number of sprints $(F=11.22, P=0.004)$ and total work $(F=8.11, P=0.01)$ performed during the RSA test.

Isolated 10-s sprint peak power output and TS performance increased $(P<0.01)$ similarly in RSH and RSN (Table 3). For instance, the average power output during all TS improved similarly by $11 \% \pm 9 \%$ and $15 \% \pm 7 \%$, respectively.

Muscle oxygenation. Successive values in $\Delta[\mathrm{tHb}]$ during the RSA test are displayed in Figure 3. After training,

TABLE 3. Performance results before (pre) and after (post) repeated double-poling sprint training in RSN or in RSH.

\begin{tabular}{|c|c|c|c|c|}
\hline & \multicolumn{2}{|c|}{ RSN } & \multicolumn{2}{|c|}{ RSH } \\
\hline & Pretraining & Posttraining & Pretraining & Posttraining \\
\hline Single 10-s sprint average power (W) & $441 \pm 153$ & $539 \pm 158^{*}$ & $421 \pm 122$ & $534 \pm 123^{*}$ \\
\hline RSA peak power (W) & $437 \pm 156$ & $532 \pm 158^{\star}$ & $415 \pm 117$ & $528 \pm 121^{*}$ \\
\hline RSA best average power (W) & $365 \pm 124$ & $440 \pm 128^{\star}$ & $350 \pm 91$ & $437 \pm 98^{*}$ \\
\hline RSA mean power of all sprints (W) & $315 \pm 107$ & $376 \pm 111^{*}$ & $308 \pm 94$ & $352 \pm 78^{*}$ \\
\hline RSA total work of all sprints (kJ) & $33.1 \pm 11.9$ & $42.0 \pm 16.6$ & $30.6 \pm 12.8$ & $58.8 \pm 24.4^{\star, \star \star}$ \\
\hline $\mathrm{RSA}[\mathrm{La}]\left(\mathrm{mmol} \cdot \mathrm{L}^{-1}\right)$ & $11.0 \pm 2.5$ & $12.6 \pm 2.1$ & $11.1 \pm 2.0$ & $12.3 \pm 2.9$ \\
\hline $\mathrm{RSA} \mathrm{SO}_{2}(\%)$ & $93.2 \pm 0.8$ & $93.6 \pm 1.4$ & $94.2 \pm 1.2$ & $93.5 \pm 2.2$ \\
\hline RSA blood pH & $7.23 \pm 0.04$ & $7.22 \pm 0.06$ & $7.21 \pm 0.05$ & $7.22 \pm 0.04$ \\
\hline $3 \times 3$ average power $(\mathrm{W})$ & $218 \pm 62$ & $239 \pm 64^{*}$ & $219 \pm 49$ & $240 \pm 46^{\star}$ \\
\hline $3 \times 3$ average power during first minute $(\mathrm{W})$ & $239 \pm 64$ & $264 \pm 69^{*}$ & $243 \pm 49$ & $272 \pm 50^{*}$ \\
\hline $3 \times 3$ stroke rate (per minute) & $60 \pm 4$ & $57 \pm 4$ & $64 \pm 8$ & $62 \pm 9$ \\
\hline $3 \times 3[\mathrm{La}]\left(\mathrm{mmol} \cdot \mathrm{L}^{-1}\right)$ & $11.7 \pm 3.0$ & $11.9 \pm 2.8$ & $13.1 \pm 2.2$ & $14.1 \pm 2.9$ \\
\hline
\end{tabular}

RSA parameters were measured immediately after repeated-sprint test.

$3 \times 3$, three times 3-min all-out TS; [La], blood lactate concentration.

${ }^{\star} P<0.01$ for difference with pretraining.

${ }^{\star *} P<0.05$ for difference with RSN. 

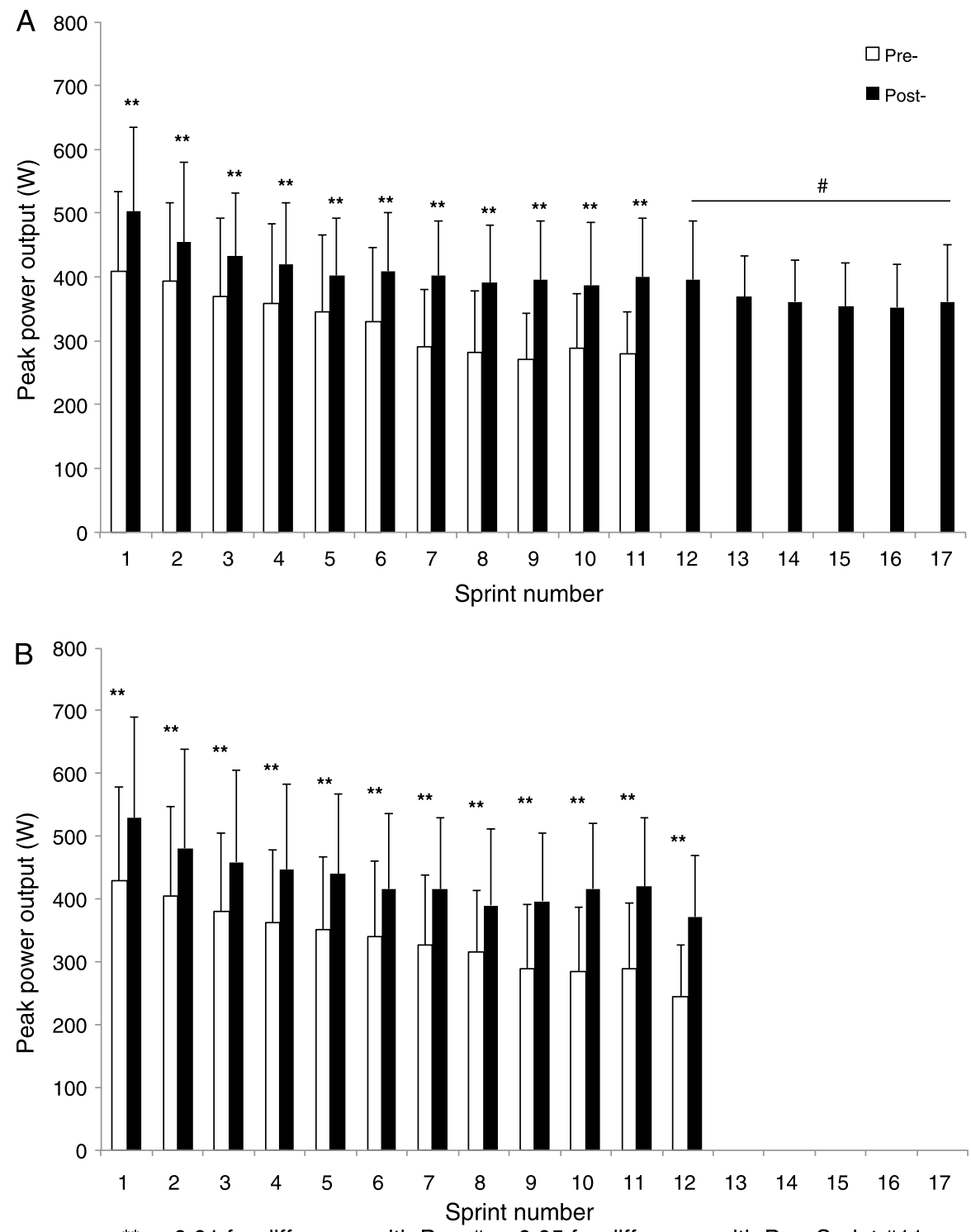

FIGURE 2-Peak power output (W) in successive sprints during the repeated double-poling sprint test before (pre) and after (post) specific repeated double-poling sprint training in hypoxia (RSH) (A) or in normoxia (RSN) (B).

$\Delta[\mathrm{tHb}]_{\mathrm{av}}$ increased to a greater extent $(F=35.9, P<0.001)$ in RSH (from $212 \% \pm 12 \%$ to $713 \% \pm 21 \%$, pretraining to posttraining) than in RSN (from $186 \% \pm 19 \%$ to $361 \% \pm$ $30 \%$, pretraining to posttraining) (Fig. 3B). From pretraining to posttraining, $\Delta[\mathrm{HHb}]_{\mathrm{av}}$ increased significantly in $\mathrm{RSH}$ $(+225 \%, P<0.01)$ but decreased in RSN $(-27 \%, P<0.01)$. There was a significant training group-time interaction for $\Delta[\mathrm{HHb}]_{\mathrm{av}}(F=551.8, P<0.001)$.

Surface EMG. From pretraining to posttraining, the mean amplitude of the activity of all muscles taken together (expressed as the sum of the percentage of MVC of all muscles) did not change in RSA test neither in RSH $(119.1 \% \pm 22.2 \%$ vs $121.6 \% \pm 17.7 \%$, NS) nor in RSN $(118.2 \% \pm 27.7 \%$ vs $121.3 \% \pm 76.2 \%$, NS). Similarly, the mean amplitude (all 3-min bouts taken together) did not change during TS neither in RSH $(205.8 \% \pm 76.0 \%$ vs
$216.9 \% \pm 69.6 \%$, NS) nor in RSN $(204.0 \% \pm 49.8 \%$ vs $176.0 \% \pm 34.0 \%, \mathrm{NS})$.

\section{DISCUSSION}

The main finding of the present investigation was that with competitive XC skiers, repeated double-poling sprint training in hypoxia (involving large upper body muscle groups) resulted in greater improvement in repeated-sprint performance than analogous normoxic training (RSN). Secondly, the amplitude of variations in blood perfusion during sprints increased to a greater extent in RSH than in RSN. Lastly, these differences were specific to repeated sprinting, i.e., performance in connection with an aerobic simulated team sprint was enhanced to a similar degree by RSH and RSN. 


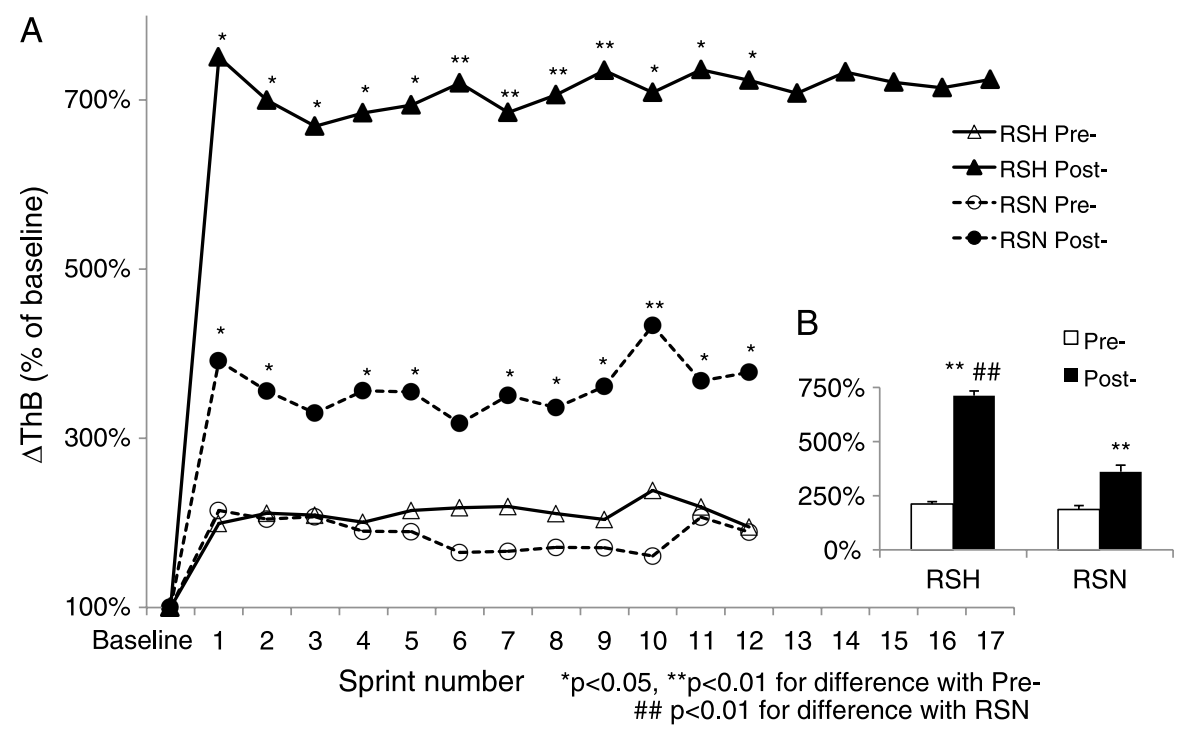

FIGURE $3-\Delta[\mathrm{tHb}]$, successive values in total hemoglobin concentrations' amplitude during sprints (expressed in percent compared with the resting baseline set to $100 \%$ ) measured by NIRS (A), and $\Delta[\mathrm{tHb}]_{\mathrm{av}}$, average of all amplitude values during the repeated double-poling sprint test to exhaustion (B) before (pre) and after (post) the specific repeated double-poling sprint training in hypoxia (RSH) or in normoxia (RSN).

The present study confirms the potential of RSH as an innovative hypoxic training method in line with similar benefits and underpinning mechanisms recently reported in cyclists (15). Interestingly, RSH in XC skiers seemed to delay fatigue during an RSA test, with 58\% more sprints performed after training. This compares well with the $38 \%$ increase in sprint repetition reported in cycling in a strictly similar RSA test (10-s sprints, 20-s recovery period) (15). Whereas the cyclists in the latter performed sprints to exhaustion, XC skiers in this study were stopped during RSA when maximal power output during sprinting did not reach $70 \%$ of the best sprint anymore. In fact, this threshold was similar in the cycling study, so it can be argued that RSH in $\mathrm{XC}$ skiers was more efficient than that in cyclists. This was expected because we hypothesized that the maximal solicitation of FT during sprints in RSH would challenge adaptive mechanisms at the muscular level for sufficient $\mathrm{O}_{2}$ extraction/delivery. With a higher proportion of FT in upper arm muscles $(32,34)$ contributing greatly to power production during double poling (24), the mechanism of compensatory vasodilation particularly important in FT when $\mathrm{O}_{2}$ tension falls (10) is enhanced after RSH. This may thus explain why fatigue was more delayed during RSA in XC skiers than in cyclists.

However, the NIRS measurement methods allowed to record local variations in total hemoglobin/myoglobin content $(\Delta[\mathrm{tHb}])$ in the muscle, thus reflecting blood perfusion and possibly blood flow variations $(20,39)$. So, this method provided only an indirect estimation of muscle perfusion variations (and thus blood flow), and our interpretations should be considered with care. Nevertheless, the gains in the amplitude of the variations after RSH may be more important when measured here in $m$. triceps brachii compared with $m$. vastus lateralis we reported recently (15). Eventually, the latter is in accordance with our hypothesis that performing RSH in activities soliciting more FT fibers (e.g., cross-country double poling with the upper body comprising more FT (22)) would potentiate larger benefits in RSA performance gains. Research on isolated animal muscles containing almost exclusively FT might help elucidate the adaptive mechanisms involved in greater detail.

We hypothesized that XC double poling would substantially benefit from fiber-type selective mechanisms, allowing better $\mathrm{O}_{2}$ extraction by FT after RSH because upper body muscles are largely contributing to power production (24).

Mechanistically, the increase in cardiac output $(\dot{Q})$ tightly matches the $\mathrm{O}_{2}$ delivery by elevating blood flow, and interestingly, the rise in $\dot{Q}$ is mainly regulated by peripheral vasodilation changes (with less importance of HR increase) (2). Then, high-intensity intermittent training (e.g., knee extension) was reported to increase the capacity for maximal exercise vasodilation by $20 \%-30 \%$ (9). The latter may in turn partly explain the twofold higher amplitude of blood perfusion variations observed after RSN (Fig. 3B). Whereas vascular conductance may not be a limitation to $\mathrm{O}_{2}$ transport to the thigh muscle (1), arm muscles may incur a blood flow limitation when other muscles are involved during the exercise (41) such as during intense double-poling exercise known to greatly recruit not only upper body muscles but also trunk and leg muscles (24). This hypothesis is in accordance with the vasoconstrictor signals opposing the vasodilatory metabolites observed during upright whole body exercise (8).

Furthermore, hypoxia per se was shown to increase blood flow and vascular conductance during exercise with a regulation by the carotid chemoreceptors (37). Consequently, the threefold increase in arm muscle (triceps brachii) perfusion variations during repeated sprinting observed after RSH (Fig. 3B) indicates the cumulative role of hypoxia, possibly challenging to a further extent the adaptations 
observed in RSN. Accordingly, a recent study reported a $16 \%$ increase in arm blood flow and $6 \%$ in arm $\mathrm{O}_{2}$ extraction after prolonged low-intensity $\left(60 \%\right.$ of $\left.\mathrm{HR}_{\max }\right) \mathrm{XC}$ ski training (5). Because FT have been shown to optimally increase their fraction $\mathrm{O}_{2}$ extraction during intensive exercise (28), RSH may thus recruit FT maximally and maximize vascular conductance and $\mathrm{O}_{2}$ extraction to delay fatigue during subsequent RSA in normoxia.

In addition, we stated that the exercise/rest ratio plays an important role in the putative benefits after RSH (29) because it modifies the energetic contribution of glycolysis and the FT recruitment/activation during high-intensity exercise $(3,38)$. Then the rate of postsprint phosphocreatine ( $\mathrm{PCr})$ resynthesis is paramount for maintaining power production during sustained RSA (18). If RSH allows increasing muscle blood perfusion and vascular conductance as mentioned previously, it may in turn also elevate locally microvascular $\mathrm{PO}_{2}$ that is known to reduce $\mathrm{PCr}$ breakdown and speed up $\mathrm{PCr}$ resynthesis (21). Because hypoxia per se was shown to modulate PCr recovery (23), RSH may accordingly optimally delay fatigue during RSA if an optimal exercise/rest ratio is found. In addition, the lack of differences in both groups from pretraining to posttraining in the surface EMG measurements supports the hypothesis of improved metabolic glycolytic activity rather than higher power outputs due to greater recruitment of motor units. Besides, the lack of difference in the aerobic team sprint test between RSH and RSN illustrated that a prolonged intense effort (that is more aerobic by definition) does not benefit to the same extent from RSH with the lesser activation of FT. However, we point out that both isolated sprint and team sprint were improved similarly after RSH and RSN and that there is no downregulation in maximal anaerobic alactic or aerobic exercises after RSH.

Interestingly, a recent update of the current panorama of the hypoxic training methods now distinguishes RSH from traditional IHT methods, predominantly on the basis of other expected adaptive mechanisms (e.g., improvement of the muscle oxidative or buffering capacities) (30). Indeed, a current review questioned the efficiency of IHT and highlighted

\section{REFERENCES}

1. Andersen P, Saltin B. Maximal perfusion of skeletal muscle in man. J Physiol. 1985;366:233-49.

2. Bada AA, Svendsen JH, Secher NH, Saltin B, Mortensen SP. Peripheral vasodilatation determines cardiac output in exercising humans: insight from atrial pacing. $J$ Physiol. 2012;590(Pt 8):2051-60.

3. Balsom PD, Seger JY, Sjodin B, Ekblom B. Physiological responses to maximal intensity intermittent exercise. Eur J Appl Physiol Occup Physiol. 1992;65(2):144-9.

4. Borg GA. Psychophysical bases of perceived exertion. Med Sci Sports Exerc. 1982;14(5):377-81.

5. Boushel R, Ara I, Gnaiger E, et al. Low-intensity training increases peak arm $\mathrm{VO}_{2}$ by enhancing both convective and diffusive $\mathrm{O}_{2}$ delivery. Acta Physiol (Oxf). 2014;211(1):122-34. that of 20 studies involving IHT, only four bring additional benefits in performance-related variables compared with similar training in normoxia (14).

Finally, a recent study investigated different severity of the hypoxic stress (e.g., at simulated altitude of 2000, 3000, and $4000 \mathrm{~m}$ ), highlighting that "the higher may not be the better" in the training quality for RSH to improve repeatedsprint performance (19). Further studies could consequently focus on the exercise/rest ratio to optimize RSH benefits and find the optimal balance between maximal exercise intensity and the added hypoxic stress. So far, we can only observe that the chosen simulated altitude (e.g., normobaric hypoxia) of $3000 \mathrm{~m}$ seems to be providing a stimulus large enough for inducing additional benefits in RSH. One cannot rule out that in hypobaric hypoxia, the adaptations would be even higher (31) because we reported different responses in nitrosative and oxidative stress between normobaric and hypobaric hypoxia at $3000 \mathrm{~m} \mathrm{(16).}$

In conclusion, our present findings demonstrate for the first time that greater improvement in the performance of repeated double-poling sprints can be attained with RSH compared with RSN, with larger variations in the perfusion of active muscles in the former. This adds to the accumulating evidence that RSH represents a promising and potent training strategy promoting repeated-sprint performance enhancement by adding hypoxic stress to repeated-sprint training. We propose that by including RSH sessions in their training regimen, $\mathrm{XC}$ skiers can improve their work capacity during sessions of repeated sprints. However, further examination is required to confirm that the underlying mechanisms are fiber specific and to establish definitively the potential for such training to improve performance of team sports.

We thank Elin Degerström and Andreas Kårström for their invaluable help and assistance during the experiment. The authors have no conflicts of interest, source of funding, or financial ties to disclose, and they have no current or past relationship with companies or manufacturers who could benefit from the results of the present study.

The results of the present study do not constitute endorsement by the American College of Sports Medicine.

6. Boushel R, Piantadosi CA. Near-infrared spectroscopy for monitoring muscle oxygenation. Acta Physiol Scand. 2000;168(4):615-22.

7. Buchheit M, Cormie P, Abbiss CR, Ahmaidi S, Nosaka KK, Laursen PB. Muscle deoxygenation during repeated sprint running: effect of active vs passive recovery. Int J Sports Med. 2009;30(6):418-25.

8. Calbet JA, Gonzalez-Alonso J, Helge JW, et al. Cardiac output and leg and arm blood flow during incremental exercise to exhaustion on the cycle ergometer. J Appl Physiol (1985). 2007; 103(3):969-78.

9. Calbet JA, Lundby C. Skeletal muscle vasodilatation during maximal exercise in health and disease. J Physiol. 2012;590(Pt 24): 6285-96. 
10. Casey DP, Joyner MJ. Compensatory vasodilatation during hypoxic exercise: Mechanisms responsible for matching oxygen supply to demand. $J$ Physiol. 2012;590(Pt 24):6321-6.

11. Delpy DT, Cope M. Quantification in tissue near-infrared spectroscopy. Philos T R Soc B. 1997;352(1354):649-59.

12. Dufour SP, Ponsot E, Zoll J, et al. Exercise training in normobaric hypoxia in endurance runners. I. Improvement in aerobic performance capacity. J Appl Physiol (1985). 2006;100(4):1238-48.

13. Duncan A, Meek JH, Clemence M, et al. Optical pathlength measurements on adult head, calf and forearm and the head of the newborn infant using phase resolved optical spectroscopy. Phys Med Biol. 1995;40(2):295-304.

14. Faiss R, Girard O, Millet GP. Advancing hypoxic training in team sports: from intermittent hypoxic training to repeated sprint training in hypoxia. Br J Sports Med. 2013;47(1 Suppl):i45-50.

15. Faiss R, Leger B, Vesin JM, et al. Significant molecular and systemic adaptations after repeated sprint training in hypoxia. PLoS One. 2013;8(2):e56522.

16. Faiss R, Pialoux V, Sartori C, Faes C, Deriaz O, Millet GP. Ventilation, oxidative stress, and nitric oxide in hypobaric versus normobaric hypoxia. Med Sci Sports Exerc. 2013;45(2):253-60.

17. Galvin HM, Cooke K, Sumners DP, Mileva KL, Bowtell JL. Repetead sprint training in normobaric hypoxia. Br J Sports Med. 2013;47(1 Suppl):i74-9.

18. Girard O, Mendez-Villanueva A, Bishop D. Repeated-sprint ability-part I: factors contributing to fatigue. Sports Med. 2011; 41(8):673-94.

19. Goods PS, Dawson BT, Landers GJ, Gore CJ, Peeling P. Effect of different simulated altitudes on repeat sprint performance in team sport athletes. Int J Sports Physiol Perform. 2014;9(5):857-62.

20. Hachiya T, Blaber AP, Saito M. Near-infrared spectroscopy provides an index of blood flow and vasoconstriction in calf skeletal muscle during lower body negative pressure. Acta Physiol (Oxf). 2008;193(2):117-27.

21. Haseler LJ, Hogan MC, Richardson RS. Skeletal muscle phosphocreatine recovery in exercise-trained humans is dependent on $\mathrm{O}_{2}$ availability. $J$ Appl Physiol (1985). 1999;86(6):2013-8.

22. Hautier CA, Linossier MT, Belli A, Lacour JR, Arsac LM. Optimal velocity for maximal power production in non-isokinetic cycling is related to muscle fibre type composition. Eur J Appl Physiol Occup Physiol. 1996;74(1-2):114-8.

23. Holliss BA, Fulford J, Vanhatalo A, Pedlar CR, Jones AM. Influence of intermittent hypoxic training on muscle energetics and exercise tolerance. J Appl Physiol (1985). 2013;114(5):611-9.

24. Holmberg HC, Lindinger S, Stoggl T, Eitzlmair E, Muller E. Biomechanical analysis of double poling in elite cross-country skiers. Med Sci Sports Exerc. 2005;37(5):807-18.

25. Hoppeler H, Vogt M. Muscle tissue adaptations to hypoxia. $J$ Exp Biol. 2001;204(Pt 18):3133-9.
26. Klein CS, Marsh GD, Petrella RJ, Rice CL. Muscle fiber number in the biceps brachii muscle of young and old men. Muscle Nerve. 2003;28(1):62-8.

27. Lundby C, Calbet JA, Robach P. The response of human skeletal muscle tissue to hypoxia. Cell Mol Life Sci. 2009;66(22):3615-23.

28. McDonough P, Behnke BJ, Padilla DJ, Musch TI, Poole DC. Control of microvascular oxygen pressures in rat muscles comprised of different fibre types. J Physiol. 2005;563(Pt 3):903-13.

29. Millet GP, Faiss R. Hypoxic conditions and exercise-to-rest ratio are likely paramount. Sports Med. 2012;42(12):1081-3.

30. Millet GP, Faiss R, Brocherie F, Girard O. Hypoxic training and team sports: a challenge to traditional methods? Br J Sports Med. 2013;47(1 Suppl):i6-7.

31. Millet GP, Faiss R, Pialoux V. Point: hypobaric hypoxia induces different physiological responses from normobaric hypoxia. $J \mathrm{Appl}$ Physiol (1985). 2012;112(10):1783-4.

32. Mygind E. Fibre characteristics and enzyme levels of arm and leg muscles in elite cross-country skiers. Scand J Med Sci Sports. 1995;5(2):76-80.

33. Puype J, Van Proeyen K, Raymackers JM, Deldicque L, Hespel P. Sprint interval training in hypoxia stimulates glycolytic enzyme activity. Med Sci Sports Exerc. 2013;45(11):2166-74.

34. Sanchis-Moysi J, Idoate F, Olmedillas H, et al. The upper extremity of the professional tennis player: muscle volumes, fiber-type distribution and muscle strength. Scand J Med Sci Sports. 2010;20(3):524-34.

35. Sandbakk O, Holmberg HC. A reappraisal of success factors for Olympic cross-country skiing. Int J Sports Physiol Perform. 2014; 9(1):117-21.

36. Smith KJ, Billaut F. Tissue oxygenation in men and women during repeated-sprint exercise. Int J Sports Physiol Perform. 2012;7(1):59-67.

37. Stickland MK, Fuhr DP, Haykowsky MJ, et al. Carotid chemoreceptor modulation of blood flow during exercise in healthy humans. J Physiol. 2011;589(Pt 24):6219-30.

38. Tabata I, Irisawa K, Kouzaki M, Nishimura K, Ogita F, Miyachi M. Metabolic profile of high intensity intermittent exercises. Med Sci Sports Exerc. 1997;29(3):390-5.

39. Truijen J, Kim YS, Krediet CT, et al. Orthostatic leg blood volume changes assessed by near-infrared spectroscopy. Exp Physiol. 2012;97(3):353-61.

40. Vogt M, Puntschart A, Geiser J, Zuleger C, Billeter R, Hoppeler H. Molecular adaptations in human skeletal muscle to endurance training under simulated hypoxic conditions. J Appl Physiol (1985). 2001;91(1):173-82.

41. Volianitis S, Secher NH. Arm blood flow and metabolism during arm and combined arm and leg exercise in humans. $J$ Physiol. 2002;544(Pt 3):977-84.

42. Zoll J, Ponsot E, Dufour S, et al. Exercise training in normobaric hypoxia in endurance runners. III. Muscular adjustments of selected gene transcripts. J Appl Physiol (1985). 2006;100(4):1258-66. 